\title{
Approximating a solution of an equilibrium problem by Viscosity iteration involving a nonexpansive semigroup
}

\author{
Binayak S. Choudhury \& Subhajit Kundu \\ Bengal Engineering and Science University, Shibpur \\ Department of Mathematics, \\ P.O.: B. Garden, Shibpur, Howrah - 711103, West Bengal, India. \\ binayak12@yahoo.co.in, subhajit.math@gmail.com
}

\begin{abstract}
In this paper we have defined a new iteration in order to solve an equilibrium problem in Hilbert spaces. The iteration we have introduced is a viscosity type iteration and involves a semigroup of nonexpansive operators. We have established that depending on some control conditions, our iteration strongly converges to a solution of the equilibrium problem.
\end{abstract}

\section{RESUMEN}

En este artículo hemos definido una iteración nueva para resolver un problema de equilibrio en espacios de Hilbert. La iteración que introducimos es de tipo viscoso e involucra un semigrupo de operadores no expansivos. Hemos establecido que dependiendo de las condiciones de control, nuestra iteración converge fuertemente a una solución de un problema de equilibrio.

Keywords and Phrases: Equilibrium problem, Nonexpansive semigroup, Viscosity iteration, Fixed point, Weak convergence, Hilbert space.

2010 AMS Mathematics Subject Classification: 46C05, 47H10, 91B50. 


\section{Introduction and Mathematical Preliminaries}

The equilibrium problem we consider in this paper is formulated in the framework of real Hilbert spaces. This problem is a generalization of several problems in physics, optimization and economics. References [3, 9] give a good account of this feature. There are several iterative methods for obtaining solutions of this equilibrium problem in Hilbert spaces and also in the more general settings of Banach spaces [19, 10, 22]. A particular category of these iterations is viscosity iteration which was first developed by Moudafi [15] to obtain fixed points of nonexpansive mappings. Viscosity iterations have been used for solving equilibrium problems in works noted in [20, 16]. Semigroup of nonexpansive operators have been considered in the context of constructing fixed point iteration in Banach and Hilbert spaces [8, 21, 1, 6, 5, 13, 17, 11, 12, 17]. The purpose of this paper is to use nonexpansive semigroups in a viscosity iteration scheme in order to construct a two step iteration for approximating a solution of an equilibrium problem in real Hilbert spaces. Precisely, we have shown that under suitable choices of the control conditions, our iteration strongly converges to solution of the equilibrium problem.

Let $\mathrm{H}$ be a Hilbert space and $\mathrm{C}$ be a nonempty closed convex subset of $\mathrm{H}$. A mapping $\mathrm{T}: \mathrm{C} \rightarrow \mathrm{C}$ is said to be a nonexpansive mapping if for all $x, y \in \mathrm{C}$

$$
\|T x-T y\| \leq\|x-y\| \text {. }
$$

A mapping $f: C \rightarrow C$ is said to be a $\theta$ contraction if for each $x, y \in C$,

$$
\|f x-f y\| \leq \theta\|x-y\| \text { when } 0<\theta<1
$$

For any $x \in H$, the metric projection $P_{C}$ from $H$ into $C$ is defined as

$$
P_{C} x=\left\{z \in C:\|z-x\|=\inf _{y \in C}\|y-x\|\right\} .
$$

Obviously, $\left\|x-P_{C} x\right\| \leq\|x-y\|$. It is well known that $P_{C}$ is a firmly nonexpansive mapping from $\mathrm{H}$ onto $\mathrm{C}$, that is,

$\left\|P_{C} x-P_{C} y\right\|^{2} \leq\left\langle P_{C} x-P_{C} y, x-y\right\rangle$ for all $x, y \in H$. $P_{C}$ is also nonexpansive mapping from $H$ onto $\mathrm{C}$.

The set of fixed point of an operator $T$ from $H$ to $H$ is denoted by $F i x(T)$, that is, Fix $(T)=\{x \in H$ : $\mathrm{T} x=x\}$.

A family $S=(T(s))_{s \geq 0}$ is a nonexpansive semigroup on $\mathrm{H}$ if it satisfies the following conditions:

(A1) $\mathrm{T}(0) x=x$ for all $x \in H$,

(A2) $\mathrm{T}(\mathrm{s}+\mathrm{t})=\mathrm{T}(\mathrm{s}) \mathrm{T}(\mathrm{t})$ for all $\mathrm{s}, \mathrm{t} \geq 0$,

(A3) $\|\mathrm{T}(\mathrm{s}) \mathrm{x}-\mathrm{T}(\mathrm{s}) \mathrm{y}\| \leq\|x-y\|$ for all $x, y \in H$ and $s \geq 0$,

(A4) for all $x \in H, s \rightarrow T(s) x$ is continuous.

A sequence $\left\{x_{n}\right\}$ of elements of a Banach space $X$ is said to converge weakly to an element $x \in X$ if $f\left(x_{k}\right) \rightarrow f(x)$ as $k \rightarrow \infty$ for all $f \in X^{\prime}$ where $f$ is a continuous linear functional from $X$ to $R$ or $C$ where $R$ is the set of real numbers and $C$ is the set of complex number, and $X^{\prime}$ is the dual of $X$. A sequence $\left\{x_{n}\right\}$ is said to have a weak limit point $l$ if there exists a subsequence $\left\{x_{n_{k}}\right\}$ of $\left\{x_{n}\right\}$ which converges weakly to $l$. 
We denote $w_{\omega}\left(x_{n}\right)$ as the set of all weak limit point of $\left\{x_{n}\right\}$ and $w_{s}\left(x_{n}\right)$ as the set of all strong limit point of $\left\{x_{n}\right\}$.

We denote the set of fixed point of $T(s)$ by $\operatorname{Fix}(T(s))$. The set of all common fixed points of $S$ is denoted by $\operatorname{Fix}(S)$. So $\operatorname{Fix}(S)=\cap_{\mathrm{s} \geq 0} \operatorname{Fix}(\mathrm{T}(\mathrm{s}))$.

Baillon proved the following nonlinear ergodic theorem:

Theorem 1.1. [1] If $T$ is a nonexpansive mapping from $C$ into itself such that $F i x(T) \neq \phi$ and $x \in C$, then $\frac{1}{n} \sum_{k=0}^{n-1} T^{k} x$ converges weakly to a fixed point of $T$.

Later Baillon and Brezis proved the following theorem for semigroup of nonexpansive operator:

Theorem 1.2. [2] If $S=(T(s))_{s \geq 0}$ is a nonexpansive semigroup on $C$, then $\left\{\frac{1}{t} \int_{0}^{t} T(s) x_{t} d s\right\}_{t}>0$, $t \in(0,1), s \in R^{+}$, where $R^{+}$is the set of positive real numbers, converges weakly to a common fixed point of $S$.

Let $\mathrm{F}: \mathrm{C} \times \mathrm{C} \rightarrow \mathrm{R}$ be a bifunction where $\mathrm{R}$ is the set of real numbers. The equilibrium problem is to find some $x \in \mathrm{C}$ such that

$$
F(x, y) \geq 0 \text {, for all } y \in C \text {. }
$$

The set of solutions of (1.4) is denoted by $E P(F)$, that is, $E P(F)=\{x \in C: F(x, y) \geq 0$ for all $y \in C\}$. In the equilibrium problem for the bifunction $F$ from $C \times C \rightarrow R$, we assume that $F$ satisfies following conditions:

(C1) $F(x, x)=0$ for all $x \in C$,

(C2) $F$ is monotone, that is, $F(x, y)+F(y, x) \leq 0$,

(C3) for each $x, y, z \in C$,

$$
\lim _{t \rightarrow 0^{+}} F(t z+(1-t) x, y) \leq F(x, y)
$$

(C4) for each $x \in C, y \rightarrow F(x, y)$ is convex and lower semicontinuous.

Lemma 1.1. [9] Let $\mathrm{C}$ be a nonempty closed convex subset of $\mathrm{H}$ and let $\mathrm{F}$ be a bifunction from $\mathrm{C} \times \mathrm{C}$ into $\mathrm{R}$ satisfying conditions (C1)- (C4). Then for any $r>0$ and $x \in \mathrm{H}$ there exists $z \in \mathrm{C}$ such that

$$
\mathrm{F}(z, y)+\frac{1}{\mathrm{r}}\langle y-z, z-x\rangle \geq 0 \text { for all } y \in C .
$$

Further, if $\mathrm{T}_{\mathrm{r}} x=\left\{z \in \mathrm{C}: \mathrm{F}(z, y)+\frac{1}{\mathrm{r}}\langle y-z, z-x\rangle \geq 0\right.$ for all $\left.y \in C\right\}$ then the following hold:

(1) $T_{r}$ is single valued,

(2) $T_{r}$ is firmly nonexpansive, that is, for any $x, y \in H$

(3) $\operatorname{Fix}\left(T_{r}\right)=E P(F)$,

$$
\left\|T_{r} x-T_{r} y\right\|^{2} \leq\left\langle T_{r} x-T_{r} y, x-y\right\rangle,
$$

(4) $\mathrm{EP}(\mathrm{F})$ is closed and convex.

Lemma 1.2. [4] Let $\mathrm{C}$ be a nonempty closed convex subset of a real Hilbert space $H$. Given $z \in \mathrm{H}$ and $x \in \mathrm{C}$, the inequality $\langle x-z, y-x\rangle \geq 0$, for all $y \in \mathrm{C}$ holds if and only if $x=\mathrm{P}_{\mathrm{C}} z$, where $\mathrm{P}_{\mathrm{C}}$ denotes the metric projection from $\mathrm{H}$ onto $\mathrm{C}$.

Lemma 1.3. [14] Let $\left\{a_{n}\right\},\left\{b_{n}\right\}$ and $\left\{c_{n}\right\}$ be three nonnegative real sequences satisfying

$$
a_{n+1} \leq\left(1-\lambda_{n}\right) a_{n}+b_{n}+c_{n}, \quad n \geq n_{0},
$$

where $n_{0}$ is some nonnegative integer, $\lambda_{n} \in[0,1], \sum_{n=1}^{\infty} \lambda_{n}=\infty, b_{n}=o\left(\lambda_{n}\right)$, and $\sum_{n=1}^{\infty} c_{n}<\infty$. 
Then $a_{n} \rightarrow 0$ as $n \rightarrow \infty$.

Lemma 1.4. [18] Let $\mathrm{C}$ be a nonempty bounded closed convex subset of a Hilbert space $\mathrm{H}$ and let $(T(s))_{s>0}$ be a nonexpansive semigroup on $C$. Then for every $h \geq 0$, $\lim _{t \rightarrow \infty} \sup _{x \in C}\left\|\frac{1}{t} \int_{0}^{t} T(s) x d s-T(h) \frac{1}{t} \int_{0}^{t} T(s) x d s\right\|=0$.

Lemma 1.5. [4] Let $X$ be a uniformly convex Banach space, $C$ be a nonempty closed convex subset of $\mathrm{X}$ and $\mathrm{T}: \mathrm{C} \rightarrow \mathrm{X}$ be a nonexpansive mapping. Then, the mapping ( $\mathrm{I}-\mathrm{T}$ ) is demiclosed on $C$, that is, if $\left\{x_{n}\right\}$ is weakly convergent to $x$ and $\left\{(I-T) x_{n}\right\}$ is strongly convergent to $y$, then $(\mathrm{I}-\mathrm{T}) \mathrm{x}=\mathrm{y}$.

Lemma 1.6. [8] Let us suppose (C1)-(C4) hold. Let $x, y \in H, r_{1}, r_{2}>0$. Then $\left\|\mathrm{T}_{\mathrm{r}_{2}} \mathrm{y}-\mathrm{T}_{\mathrm{r}_{1}} x\right\| \leq\|y-x\|+\left|\frac{\mathrm{r}_{2}-\mathrm{r}_{1}}{\mathrm{r}_{2}}\right|\left\|\mathrm{T}_{\mathrm{r}_{2}} \mathrm{y}-\mathrm{y}\right\|$.

Lemma 1.7. [23] Let $\left\{s_{n}\right\}$ be a sequence of nonnegative real numbers satisfying $s_{n+1} \leq\left(1-\gamma_{n}\right) s_{n}+\sigma_{n}+\delta_{n}$, for all $n \geq 0$, where $\left\{\gamma_{n}\right\}$ is a sequence in $(0,1)$ and $\left\{\sigma_{n}\right\},\left\{\delta_{n}\right\}$ are sequences of real numbers such that

(a) $\lim _{n \rightarrow \infty} \gamma_{n}=0$ and $\sum_{n=0}^{\infty} \gamma_{n}=\infty$,

(b) $\limsup _{n \rightarrow \infty} \frac{\sigma_{n}}{\gamma_{n}} \leq 0$,

(c) $\delta_{n} \geq 0$ and $\sum_{n=1}^{\infty} \delta_{n}<\infty$.

Then $\left\{s_{n}\right\}$ converges to zero.

The following lemma is a well known result of functional analysis.

Lemma 1.8. Let $X$ be a reflexive Banach space. Then every bounded sequence in $X$ has a weakly convergent subsequence.

\section{Main Result}

Theorem 2.1. Let $S=(T(s))_{s \geq 0}$ be a nonexpansive semigroup on a real Hilbert space $H$. Let $\mathrm{f}: \mathrm{H} \rightarrow \mathrm{H}$ be a $\theta$-contraction, with $0<\theta<1$. Let $\mathrm{F}: \mathrm{H} \times \mathrm{H} \rightarrow \mathrm{R}$ be a mapping satisfying hypothesis (C1)-(C4). Assume that $\operatorname{Fix}(\mathrm{S}) \cap \mathrm{EP}(\mathrm{F}) \neq \phi$. Let $x_{0} \in \mathrm{H},\left\{z_{\mathrm{n}}\right\} \subset \mathrm{H}$ and $\left\{x_{n}\right\} \subset \mathrm{H}$ be the sequences generated by

$$
\left\{\begin{array}{l}
x_{n+1}=\beta_{n} x_{n}+\left(1-\beta_{n}\right) \frac{1}{s_{n}} \int_{0}^{s_{n}} T(s) y_{n} d s, \\
y_{n}=\alpha_{n} f\left(x_{n}\right)+\left(1-\alpha_{n}\right) z_{n}, \\
F\left(z_{n}, y\right)+\frac{1}{r_{n}}\left\langle y-z_{n}, z_{n}-x_{n}\right\rangle \geq 0, \text { for all } y \in H
\end{array}\right.
$$

where $\left\{\alpha_{n}\right\},\left\{\beta_{n}\right\},\left\{s_{n}\right\}$ and $\left\{r_{n}\right\}$ satisfy the following conditions. 
(i) $\alpha_{n} \in[0,1], \lim _{n \rightarrow \infty} \alpha_{n}=0, \sum_{n=1}^{\infty} \alpha_{n}=\infty$ and $\sum_{n=1}^{\infty}\left|\alpha_{n}-\alpha_{n-1}\right|<\infty$,

(ii) $\lim _{n \rightarrow \infty} s_{n}=\infty$ and $\lim _{n \rightarrow \infty} \frac{\left|s_{n}-s_{n-1}\right|}{s_{n}} \frac{1}{\alpha_{n}}=0$,

(iii) $\liminf _{n \rightarrow \infty} r_{n}>0, \sum_{n=0}^{\infty}\left|r_{n+1}-r_{n}\right|<\infty$,

(iv) $0<\beta_{n} \leq d<1, \lim _{n \rightarrow \infty} \beta_{n}=0, \sum_{n=0}^{\infty}\left|\beta_{n+1}-\beta_{n}\right|<\infty$.

Then $\left\{x_{n}\right\}$ converges strongly to a point $p \in \operatorname{Fix}(S) \cap E P(F)$.

Proof. Here $P_{F i x(S) \cap E P(F)} f$ is a mapping of $H$ into Fix $(S) \cap E P(F) \subset H$ such that

$\left\|P_{F i x(S) \cap E P(F)} f(x)-P_{F i x(S) \cap E P(F)} f(y)\right\|$

$\leq\|f(x)-f(y)\| \leq \theta\|x-y\|$.

Therefore $P_{F i x(S) \cap E P(F)} f$ is a contractive mapping and hence, by Banach's contraction principle, there exists a unique element $p \in F i x(S) \cap E P(F)$ such that $p=P_{F i x(S) \cap E P(F)} f(p)$. Now, for this $p \in \operatorname{Fix}(S) \cap \operatorname{EP}(F), n \geq 0$, we have,

$\left\|x_{n+1}-p\right\|$

$=\left\|\beta_{n} x_{n}+\left(1-\beta_{n}\right) \frac{1}{s_{n}} \int_{0}^{s_{n}} T(s) y_{n} d s-p\right\|$

$=\left\|\beta_{n} x_{n}+\left(1-\beta_{n}\right) \frac{1}{s_{n}} \int_{0}^{s_{n}} T(s) y_{n} d s-\frac{1}{s_{n}} \int_{0}^{s_{n}} T(s) p d s\right\|$

$=\left\|\beta_{n}\left(x_{n}-p\right)+\left(1-\beta_{n}\right) \frac{1}{s_{n}} \int_{0}^{s_{n}}\left(T(s) y_{n}-T(s) p\right) d s\right\|$

$\leq \beta_{n}\left\|x_{n}-p\right\|+\left(1-\beta_{n}\right)\left\|y_{n}-p\right\|$.

Now, $\left\|y_{n}-p\right\|=\left\|\alpha_{n} f\left(x_{n}\right)+\left(1-\alpha_{n}\right) z_{n}-p\right\|$ $\leq \alpha_{n}\left\|f\left(x_{n}\right)-f(p)\right\|+\alpha_{n}\|f(p)-p\|+\left(1-\alpha_{n}\right)\left\|z_{n}-p\right\|$.

Since by Lemma 1.1 we have $z_{n}=T_{r_{n}} x_{n}, p=T_{r_{n}} p$ it follows that for all $n \geq 0,\left\|z_{n}-p\right\|=$ $\left\|\mathrm{T}_{\mathrm{r}_{n}} x_{\mathrm{n}}-\mathrm{T}_{\mathrm{r}_{\mathrm{n}}} \mathrm{p}\right\| \leq\left\|x_{\mathrm{n}}-\mathrm{p}\right\|$.

Therefore, for all $n \geq 0,\left\|y_{n}-p\right\| \leq \alpha_{n} \theta\left\|x_{n}-p\right\|+\alpha_{n}\|f(p)-p\|+\left(1-\alpha_{n}\right)\left\|x_{n}-p\right\|$

$$
=\left\{1-\alpha_{n}(1-\theta)\right\}\left\|x_{n}-p\right\|+\alpha_{n}(1-\theta) \frac{\|f(p)-p\|}{1-\theta} \text {. }
$$

Therefore, for all $n \geq 0,\left\|y_{n}-p\right\| \leq \max \left\{\left\|x_{n}-p\right\|, \frac{\|f(p)-p\|}{1-\theta}\right\}$.

Therefore, for all $n \geq 0,\left\{y_{n}\right\}$ is bounded. So $\left\{z_{n}\right\}$ and $\left\{f\left(x_{n}\right)\right\}$ are also bounded.

Hence for all $n \geq 0,\left\|x_{n+1}-p\right\| \leq \max \left\{\left\|x_{n}-p\right\|, \frac{\|f(p)-p\|}{1-\theta}\right\}$.

Proceeding in the same way we get for all $n \geq 0,\left\|x_{n+1}-p\right\| \leq \max \left\{\left\|x_{0}-p\right\|, \frac{\|f(p)-p\|}{1-\theta}\right\}$. Therefore, $\left\{x_{n}\right\}$ is bounded.

Again, for all $n \geq 0$, we have, $x_{n+1}=\beta_{n} x_{n}+\left(1-\beta_{n}\right) u_{n}$ where $u_{n}=\frac{1}{s_{n}} \int_{0}^{s_{n}} T(s) y_{n} d s$.

Again, for all $n \geq 0,\left\|u_{n}-p\right\|=\left\|\frac{1}{s_{n}} \int_{0}^{s_{n}} T(s) y_{n} d s-\frac{1}{s_{n}} \int_{0}^{s_{n}} T(s) p d s\right\|$

So $\left\{u_{n}\right\}$ is also bounded.

$$
\leq\left\|y_{n}-p\right\| \leq \max \left\{\left\|x_{n}-p\right\|, \frac{\|f(p)-p\|}{1-\theta}\right\} \text {. }
$$


Now, for all $n \geq 0, x_{n+1}-x_{n}=\beta_{n} x_{n}+\left(1-\beta_{n}\right) u_{n}-\beta_{n-1} x_{n-1}-\left(1-\beta_{n-1}\right) u_{n-1}$.

Therefore, for all $n \geq 0,\left\|x_{n+1}-x_{n}\right\|$

$=\left\|\left(1-\beta_{n}\right)\left(u_{n}-u_{n-1}\right)-\left(\beta_{n}-\beta_{n-1}\right) u_{n-1}+\beta_{n}\left(x_{n}-x_{n-1}\right)+\left(\beta_{n}-\beta_{n-1}\right) x_{n-1}\right\|$

$\leq\left(1-\beta_{n}\right)\left\|u_{n}-u_{n-1}\right\|+\left|\beta_{n}-\beta_{n-1}\right|\left\{\left\|u_{n-1}\right\|+\left\|x_{n-1}\right\|\right\}+\beta_{n}\left\|x_{n}-x_{n-1}\right\|$.

Now, for all $n \geq 0,\left\|u_{n}-u_{n-1}\right\|$

$=\left\|\frac{1}{s_{n}} \int_{0}^{s_{n}} \mathrm{~T}(s) y_{n} d s-\frac{1}{s_{n-1}} \int_{0}^{s_{n-1}} \mathrm{~T}(s) y_{n-1} d s\right\|$

$=\left\|\frac{1}{s_{n}} \int_{0}^{s_{n}}\left(T(s) y_{n}-T(s) y_{n-1}\right) d s+\left(\frac{1}{s_{n}}-\frac{1}{s_{n-1}}\right) \int_{0}^{s_{n-1}} T(s) y_{n-1} d s+\frac{1}{s_{n}} \int_{s_{n-1}}^{s_{n}} T(s) y_{n-1} d s\right\|$.

If $p \in \operatorname{Fix}(S)$ where $S$ is the nonexpansive semigroup, then for all $n \geq 0$, we have

$$
\begin{aligned}
& \left\|u_{n}-u_{n-1}\right\| \\
= & \| \frac{1}{s_{n}} \int_{0}^{s_{n}}\left(T(s) y_{n}-T(s) y_{n-1}\right) d s+\left(\frac{1}{s_{n}}-\frac{1}{s_{n-1}}\right) \int_{0}^{s_{n-1}}\left(T(s) y_{n-1}-T(s) p\right) d s \\
& +\frac{1}{s_{n}} \int_{s_{n-1}}^{s_{n}} T(s) y_{n-1}-T(s) p d s \| \\
\leq & \left\|y_{n}-y_{n-1}\right\|+\left(\frac{2\left|s_{n}-s_{n-1}\right|}{s_{n}}\right)\left\|y_{n-1}-p\right\| .
\end{aligned}
$$

Now, for all $n \geq 0,\left\|y_{n}-y_{n-1}\right\|$

$=\left\|\alpha_{n} f\left(x_{n}\right)+\left(1-\alpha_{n}\right) z_{n}-\alpha_{n-1} f\left(x_{n-1}\right)-\left(1-\alpha_{n-1}\right) z_{n-1}\right\|$

$=\left\|\alpha_{n}\left(f\left(x_{n}\right)-f\left(x_{n-1}\right)\right)+\left(\alpha_{n}-\alpha_{n-1}\right)\left(f\left(x_{n-1}\right)-z_{n-1}\right)+\left(1-\alpha_{n}\right)\left(z_{n}-z_{n-1}\right)\right\|$.

Therefore, for all $n \geq 0$,

$$
\begin{aligned}
\left\|y_{n}-y_{n-1}\right\| & \leq \alpha_{n}\left\|f\left(x_{n}\right)-f\left(x_{n-1}\right)\right\|+\left|\alpha_{n}-\alpha_{n-1}\right|\left\|f\left(x_{n-1}\right)-z_{n-1}\right\| \\
& +\left(1-\alpha_{n}\right)\left\|z_{n}-z_{n-1}\right\| .
\end{aligned}
$$

Again, for all $\mathrm{n} \geq 0,\left\|z_{\mathrm{n}}-z_{\mathrm{n}-1}\right\|$

$\leq\left\|x_{n}-x_{n-1}\right\|+\frac{\left|r_{n}-r_{n-1}\right|}{r_{n}}\left\|z_{n}-x_{n}\right\| \quad$ (using Lemma 1.6)

We have $\liminf _{n \rightarrow \infty} r_{n}>0$. Therefore there exists $b>0$ such that $r_{n}>b$ for large $n \in N$ where $N$ is the set of positive integers. Then, for all $n \geq 0$,

$\left\|z_{n}-z_{n-1}\right\| \leq\left\|x_{n}-x_{n-1}\right\|+\frac{\left|r_{n}-r_{n-1}\right|}{b}\left\|z_{n}-x_{n}\right\|$.

Using (2.3), (2.4) in (2.2), we get, for all $n \geq 0$,

$$
\begin{aligned}
& \left\|u_{n}-u_{n-1}\right\| \\
& \leq \alpha_{n} \theta\left\|x_{n}-x_{n-1}\right\|+\left|\alpha_{n}-\alpha_{n-1}\right|\left\|f\left(x_{n-1}\right)-z_{n-1}\right\|+\left(1-\alpha_{n}\right)\left\|x_{n}-x_{n-1}\right\| \\
& +\left(1-\alpha_{n}\right) \frac{\left|r_{n}-r_{n-1}\right|}{b}\left\|z_{n}-x_{n}\right\|+\frac{2\left|s_{n}-s_{n-1}\right|}{s_{n}}\left\|y_{n-1}-p\right\| .
\end{aligned}
$$

Using (2.5) in (2.1) we get, for all $n \geq 0,\left\|x_{n+1}-x_{n}\right\|$

$\leq\left(1-\beta_{n}\right) \alpha_{n} \theta\left\|x_{n}-x_{n-1}\right\|+\left(1-\beta_{n}\right)\left|\alpha_{n}-\alpha_{n-1}\right|\left\|f\left(x_{n-1}\right)-z_{n-1}\right\|+\left(1-\beta_{n}\right)\left(1-\alpha_{n}\right)\left\|x_{n}-x_{n-1}\right\|$ $+\left(1-\beta_{n}\right)\left(1-\alpha_{n}\right) \frac{\left|r_{n}-r_{n-1}\right|}{b}\left\|z_{n}-x_{n}\right\|+\left(1-\beta_{n}\right) \frac{2\left|s_{n}-s_{n-1}\right|}{s_{n}}\left\|y_{n-1}-p\right\|+\left|\beta_{n}-\beta_{n-1}\right|\left\{\left\|u_{n-1}\right\|+\right.$ $\left.\left\|x_{n-1}\right\|\right\}+\beta_{n}\left\|x_{n}-x_{n-1}\right\|$

$=\left\{\left(1-\beta_{n}\right) \alpha_{n} \theta+\left(1-\beta_{n}\right)\left(1-\alpha_{n}\right)+\beta_{n}\right\}\left\|x_{n}-x_{n-1}\right\|+\left(1-\beta_{n}\right)\left|\alpha_{n}-\alpha_{n-1}\right|\left\|f\left(x_{n-1}\right)-z_{n-1}\right\|+(1-$ $\left.\beta_{n}\right)\left(1-\alpha_{n}\right) \frac{\left|r_{n}-r_{n-1}\right|}{b}\left\|z_{n}-x_{n}\right\|+\left(1-\beta_{n}\right) \frac{2\left|s_{n}-s_{n-1}\right|}{s_{n}}\left\|y_{n-1}-p\right\|+\left|\beta_{n}-\beta_{n-1}\right|\left\{\left\|u_{n-1}\right\|+\left\|x_{n-1}\right\|\right\}$ $=\left\{1-\alpha_{n}(1-\theta)\left(1-\beta_{n}\right)\right\}\left\|x_{n}-x_{n-1}\right\|+\left(1-\beta_{n}\right)\left|\alpha_{n}-\alpha_{n-1}\right|\left\|f\left(x_{n-1}\right)-z_{n-1}\right\|+\left(1-\beta_{n}\right)(1-$ $\left.\alpha_{n}\right) \frac{\left|r_{n}-r_{n-1}\right|}{b}\left\|z_{n}-x_{n}\right\|+\left(1-\beta_{n}\right) \frac{2\left|s_{n}-s_{n-1}\right|}{s_{n}}\left\|y_{n-1}-p\right\|+\left|\beta_{n}-\beta_{n-1}\right|\left\{\left\|u_{n-1}\right\|+\left\|x_{n-1}\right\|\right\}$ $\leq\left\{1-\alpha_{n}(1-\theta)(1-d)\right\}\left\|x_{n}-x_{n-1}\right\|+\left|\alpha_{n}-\alpha_{n-1}\right|\left\|f\left(x_{n-1}\right)-z_{n-1}\right\|+\frac{\left|r_{n}-r_{n-1}\right|}{b}\left\|y_{n}-x_{n}\right\|+$ 
$\frac{2\left|s_{n}-s_{n-1}\right|}{s_{n}}\left\|y_{n-1}-p\right\|+\left|\beta_{n}-\beta_{n-1}\right|\left\{\left\|u_{n-1}\right\|+\left\|x_{n-1}\right\|\right\}$

Let $M=\max \left\{\sup _{n \in N}\left\|f\left(x_{n-1}\right)-z_{n-1}\right\|, \sup _{n \in N}\left\|z_{n}-x_{n}\right\|, \sup _{n \in N}\left\|y_{n-1}-p\right\|, \sup _{n \in N}\left(\|\left(u_{n-1}\|+\| x_{n-1} \|\right)\right\}\right.$.

Therefore, for all $n \geq 0,\left\|x_{n+1}-x_{n}\right\|$

$\leq\left\{1-\alpha_{n}(1-\theta)(1-d)\right\}|| x_{n}-x_{n-1} \|+M\left[\left|\alpha_{n}-\alpha_{n-1}\right|+\frac{\left|r_{n}-r_{n-1}\right|}{b}+\frac{2\left|s_{n}-s_{n-1}\right|}{s_{n}}+\left|\beta_{n}-\beta_{n-1}\right|\right]$.

Let $\gamma_{n}=\alpha_{n}(1-\theta)(1-d), \sigma_{n}=2 M \frac{\left|s_{n}-s_{n-1}\right|}{s_{n}}, \delta_{n}=M\left[\left|\alpha_{n}-\alpha_{n-1}\right|+\frac{\left|r_{n}-r_{n-1}\right|}{b}+\left|\beta_{n}-\beta_{n-1}\right|\right]$.

Using the Lemma 1.7 we get

$\lim _{n \rightarrow \infty}\left\|x_{n+1}-x_{n}\right\|=0$.

Now,

$\left\|y_{n}-z_{n}\right\|=\left\|\alpha_{n} f\left(x_{n}\right)+\left(1-\alpha_{n}\right) z_{n}-z_{n}\right\|=\alpha_{n}\left\|f\left(x_{n}\right)-z_{n}\right\| \rightarrow 0$ as $n \rightarrow \infty$.

Again, for all $n \geq 0,\left\|x_{n}-u_{n}\right\|$

$=\left\|\beta_{n-1} x_{n-1}+\left(1-\beta_{n-1}\right) u_{n-1}-u_{n}\right\|$

$\leq\left\|u_{n}-u_{n-1}\right\|+\beta_{n-1}\left\|x_{n-1}-u_{n-1}\right\|$.

Since $\beta_{n} \rightarrow 0$ and $\left\|u_{n}-u_{n-1}\right\| \rightarrow 0$ as $n \rightarrow \infty$, we have,

$\left\|x_{n}-u_{n}\right\| \rightarrow 0$ as $n \rightarrow \infty$.

Also, for all $n \geq 0,\left\|z_{n}-p\right\|^{2}=\left\|T_{r_{n}} x_{n}-T_{r_{n}} p\right\|^{2}$

$$
\begin{aligned}
& \leq\left\langle T_{r_{n}} x_{n}-T_{r_{n}} p, x_{n}-p\right\rangle \quad \text { ( by Lemma 1.1) } \\
& =\left\langle z_{n}-p, x_{n}-p\right\rangle \\
& =\frac{1}{2}\left[\left\|z_{n}-p\right\|^{2}+\left\|x_{n}-p\right\|^{2}-\left\|x_{n}-z_{n}\right\|^{2}\right]
\end{aligned}
$$

Therefore, for all $\mathrm{n} \geq 0$,

$\left\|z_{n}-p\right\|^{2} \leq\left\|x_{n}-p\right\|^{2}-\left\|x_{n}-z_{n}\right\|^{2}$.

Now, for all $n \geq 0$,

$\left\|x_{n+1}-p\right\|^{2}=\left\|\beta_{n} x_{n}+\left(1-\beta_{n}\right) u_{n}-p\right\|^{2} \leq \beta_{n}\left\|x_{n}-p\right\|^{2}+\left(1-\beta_{n}\right)\left\|u_{n}-p\right\|^{2}$

Also for all $n \geq 0$,

$\left\|y_{n}-p\right\|^{2}=\left\|\alpha_{n} f\left(x_{n}\right)+\left(1-\alpha_{n}\right) z_{n}-p\right\|^{2} \leq \alpha_{n}\left\|f\left(x_{n}\right)-p\right\|^{2}+\left(1-\alpha_{n}\right)\left\|z_{n}-p\right\|^{2}$

Using (2.8) and (2.10) in (2.9), for all $n \geq 0$, we get

$\left\|x_{n+1}-p\right\|^{2}$

$\leq \beta_{n}\left\|x_{n}-p\right\|^{2}+\left(1-\beta_{n}\right)\left[\alpha_{n}\left\|f\left(x_{n}\right)-p\right\|^{2}+\left(1-\alpha_{n}\right)\left\|z_{n}-p\right\|^{2}\right] \quad\left(\right.$ since $\left.\left\|u_{n}-p\right\| \leq\left\|y_{n}-p\right\|\right)$ $\leq \beta_{n}\left\|x_{n}-p\right\|^{2}+\left(1-\beta_{n}\right) \alpha_{n}\left\|f\left(x_{n}\right)-p\right\|^{2}+\left(1-\beta_{n}\right)\left(1-\alpha_{n}\right)\left\|z_{n}-p\right\|^{2}$

$\leq\left\|x_{n}-p\right\|^{2}+\alpha_{n}\left\|f\left(x_{n}\right)-p\right\|^{2}-\left(1-\beta_{n}\right)\left\|x_{n}-z_{n}\right\|^{2} \quad[$ by $(2.8)]$

Therefore, $\left(1-\beta_{n}\right)\left\|x_{n}-z_{n}\right\|^{2} \leq\left\|x_{n}-p\right\|^{2}-\left\|x_{n+1}-p\right\|^{2}+\alpha_{n}\left\|f\left(x_{n}\right)-p\right\|^{2}$

$$
\leq\left\{\left\|x_{n}-p\right\|+\left\|x_{n+1}-p\right\|\right\}\left\|x_{n}-x_{n+1}\right\|+\alpha_{n}\left\|f\left(x_{n}\right)-p\right\|^{2}
$$

Therefore,

$\left\|x_{\mathrm{n}}-z_{\mathrm{n}}\right\| \rightarrow 0$ as $\mathrm{n} \rightarrow \infty$.

Again $\left\|z_{n}-u_{n}\right\| \leq\left\|z_{n}-x_{n}\right\|+\left\|x_{n}-u_{n}\right\|$

By (2.7) and (2.11) we have

$\left\|z_{\mathrm{n}}-\mathrm{u}_{\mathrm{n}}\right\| \rightarrow 0$ as $\mathrm{n} \rightarrow \infty$.

By (2.6), (2.7), (2.11) and (2.12) we can say that one of the sequences $\left\{x_{n}\right\},\left\{u_{n}\right\},\left\{z_{n}\right\},\left\{y_{n}\right\}$ converge if and only if the other three converge to the same limit.

By (2.6), (2.7), (2.11) and (2.12) we have 
$\omega_{w}\left(x_{n}\right)=\omega_{w}\left(u_{n}\right)=\omega_{w}\left(z_{n}\right)=\omega_{w}\left(y_{n}\right), \omega_{s}\left(x_{n}\right)=\omega_{s}\left(u_{n}\right)=\omega_{s}\left(z_{n}\right)=\omega_{s}\left(y_{n}\right)$.

Now we have, $p=P_{\text {Fix }(S) \cap \operatorname{EP}(F)} f(p)$. We shall prove that $\limsup _{n \rightarrow \infty}\left\langle f(p)-p, y_{n}-p\right\rangle \leq 0$.

We take a subsequence $\left\{y_{n_{i}}\right\}$ of $\left\{y_{n}\right\}$ such that

$\limsup _{n \rightarrow \infty}\left\langle f(p)-p, y_{n}-p\right\rangle=\lim _{i \rightarrow \infty}\left\langle f(p)-p, y_{n_{i}}-p\right\rangle$.

Since $\left\{y_{n_{i}}\right\}$ is bounded and the Hilbert space $H$ is reflexive, by Lemma 1.8, there exists a subsequence $\left\{y_{n_{i_{k}}}\right\}$ of $\left\{y_{n_{i}}\right\}$ which converges weakly to $x^{*}$. Then $x^{*}$ is also a weak limit of $\left\{x_{n}\right\}$. Let $v_{0}=P_{F i x(S) \cap \operatorname{EP}(F)} x_{0}$. Since $\left\{x_{n}\right\}$ is a bounded sequence, there exists $K$ such that $B\left(v_{0}, K\right)$ contains $\left\{x_{n}\right\}$. Moreover, $B\left(v_{0}, K\right)$ is $T(s)$-invariant for every $s \geq 0$. Therefore, we can assume that $(T(s))_{s \geq 0}$ is a nonexpansive semigroup on $\mathrm{B}\left(v_{0}, \mathrm{~K}\right)$. So by $(2.13), x^{*} \in \omega_{w}\left(u_{n}\right)=\omega_{w}\left(z_{n}\right)$. Then, from Lemma 1.4, we have, for every $h \geq 0, \lim _{n \rightarrow \infty}\left\|\frac{1}{s_{n}} \int_{0}^{s_{n}} T(s) y_{n} d s-T(h) \frac{1}{s_{n}} \int_{0}^{s_{n}} T(s) y_{n} d s\right\|=\lim _{n \rightarrow \infty} \| u_{n}-$ $T(h) u_{n} \|=0$. Therefore from Lemma 1.5, we have $x^{*} \in \operatorname{Fix}(S)$. Next we prove that $x^{*} \in \operatorname{EP}(F)$. Let $\left\{x_{n_{i_{k}}}\right\}$ be a subsequence of $\left\{x_{n_{i}}\right\}$ such that $x_{n_{i_{k}}} \rightarrow x^{*}$. From $(2.11)$ we can say that $z_{k} \rightarrow x^{*}$. Moreover, by (C2) we obtain

$\left(1 / r_{k}\right)\left\langle y-z_{k}, z_{k}-x_{k}\right\rangle \geq F\left(y, z_{k}\right), \quad$ for all $y \in H$.

By condition $(\mathrm{C} 4)$, for fixed $x \in \mathrm{H}$, the function $\mathrm{F}(x,$.$) is lower semicontinuous and convex and$ thus is weakly lower semicontinuous.

Since $z_{k} \rightarrow x$, by $(2.11)$ and the fact that $\liminf _{n \rightarrow \infty} r_{n}=b>0$, we get $\left(z_{k}-x_{k}\right) / r_{k} \rightarrow 0$. Letting $k \rightarrow \infty$, we have, $F\left(y, x^{*}\right) \leq \liminf _{k \rightarrow \infty} F\left(y, z_{k}\right) \leq 0$, for all $y \in H$.

Replacing $y$ by $y_{t}$ where $y_{t}=t y+(1-t) x^{*}, t \in[0,1]$ and using $(C 1)$ and $(C 4)$, we get $0=F\left(y_{t}, y_{t}\right) \leq t F\left(y_{t}, y\right)+(1-t) F\left(y_{t}, x^{*}\right) \leq F\left(y_{t}, y\right)$.

Therefore, $F\left(t y+(1-t) x^{*}, y\right) \geq 0, \quad t \in[0,1], y \in H$. Letting $t \rightarrow 0^{+}$and using (C3), we conclude that

$F\left(x^{*}, y\right) \geq 0, \quad y \in H$. Therefore, $x^{*} \in E P(F)$.

Since $x^{*} \in \operatorname{Fix}(S) \cap E P(F)$, from Lemma 1.2, we have, $\lim _{n \rightarrow \infty}\left\langle f(p)-p, y_{n}-p\right\rangle$

$$
\begin{aligned}
& \left.=\lim _{i \rightarrow \infty}\left\langle f(p)-p, y_{n_{i}}-p\right\rangle \quad \text { (by } \operatorname{using}(2.14)\right) \\
& =\left\langle f(p)-p, x^{*}-p\right\rangle \leq 0 .
\end{aligned}
$$

Now for $p \in \operatorname{Fix}(S) \cap E P(F)$, for all $n \geq 0$, we have,

$$
\begin{aligned}
\left\|x_{n+1}-p\right\|^{2}= & \left\|\beta_{n}\left(x_{n}-p\right)+\left(1-\beta_{n}\right)\left(u_{n}-p\right)\right\|^{2} \\
& \leq \beta_{n}\left\|x_{n}-p\right\|^{2}+\left(1-\beta_{n}\right)\left\|u_{n}-p\right\|^{2} \\
& \leq \beta_{n}\left\|x_{n}-p\right\|^{2}+\left(1-\beta_{n}\right)\left\|y_{n}-p\right\|^{2} \\
& \leq \beta_{n}\left\|x_{n}-p\right\|^{2}+\left(1-\beta_{n}\right)\left\{\left(1-\alpha_{n}\right)^{2}\left\|z_{n}-p\right\|^{2}+2 \alpha_{n}\left\langle f\left(x_{n}\right)-p, y_{n}-p\right\rangle\right\} \\
& \leq \beta_{n}\left\|x_{n}-p\right\|^{2}+\left(1-\beta_{n}\right)\left(1-2 \alpha_{n}+\alpha_{n}^{2}\right)\left\|x_{n}-p\right\|^{2}+2 \alpha_{n}\left(1-\beta_{n}\right)\left\langle f\left(x_{n}\right)-p, y_{n}-p\right\rangle \\
& \leq\left(1-2\left(1-\beta_{n}\right) \alpha_{n}\right)\left\|x_{n}-p\right\|^{2}+\alpha_{n}^{2}\left\|x_{n}-p\right\|^{2} \\
& +2 \alpha_{n}\left(1-\beta_{n}\right)\left\{\left\langle f\left(x_{n}\right)-f(p), y_{n}-p\right\rangle+\left\langle f(p)-p, y_{n}-p\right\rangle\right\} \\
& \leq\left(1-2\left(1-\beta_{n}\right) \alpha_{n}\right)\left\|x_{n}-p\right\|^{2}+\alpha_{n}^{2} M_{0}+2\left(1-\beta_{n}\right) \alpha_{n}\left(\theta\left\|x_{n}-p\right\| \cdot\left\|y_{n}-p\right\|+\eta_{n}\right) \\
& \leq\left(1-2\left(1-\beta_{n}\right) \alpha_{n}\right)\left\|x_{n}-p\right\|^{2}+\alpha_{n}^{2} M_{0}+\left(1-\beta_{n}\right) \theta \alpha_{n}\left(\left\|x_{n}-p\right\|^{2}+\left\|y_{n}-p\right\|^{2}\right)+2 \alpha_{n} \eta_{n}
\end{aligned}
$$

where $\eta_{n}=\max \left\{\left\langle f(p)-p, y_{n}-p\right\rangle, 0\right\}$ and $M_{0}=\sup _{n \geq 0}\left\{\left\|x_{n}-p\right\|^{2}+\left\|f\left(x_{n}\right)-p\right\|^{2}\right\}$.

Now, for all $n \geq 0,\left\|y_{n}-p\right\|^{2} \leq \alpha_{n}\left\|f\left(x_{n}\right)-p\right\|^{2}+\left(1-\alpha_{n}\right)\left\|z_{n}-p\right\|^{2}$ 
Received: November 2012. Accepted: September 2013.

\section{References}

[1] Baillon, J. B., Un theorème de type ergodique pour les contractions non linèaires dans un espace de Hilbert, C.R. Acad. Sci. Paris Sèr., 280 (1975), No. A-B , 1511-1514.

[2] Baillon, J. B., Brèzis, H., Une remarque sur le comportement asymptotique des semigroupes non linèaires, Houston J. Math., 2 (1976), 5-7.

[3] Blum, E., Oettli, W., From optimization and variational inequalities to equilibrium problems, Math. Student, 63 (1994), No. 1-4, 123-145.

[4] Browder, F. E., Convergence of approximants of fixed points of nonexpansive nonlinear mappings in Banach spaces, Arch. Ration. Mech. Anal., 24 (1967), 82-89.

[5] Buong, N., Strong convergence theorem for nonexpansive semigroups in Hilbert space, Nonlinear Anal., 72 (2010), No. 12, 4534-4540.

[6] Ceng, L. C., Schaible, S., Yao, J. C., Approximate solutions of variational inequalities on sets of common fixed points of a one parameter semigroup of nonexpansive mappings, J.Optim.Theory.Appl., 143 (2009), No. 2, 245-263.

[7] Chen, R., Song, Y., Convergence to common fixed point of nonexpansive semigroups, J.Comp.Appl.Math., 200 (2007), No. 2, 566-575.

[8] Cianciaruso, F., Marino, G., Mugila, L., Iterative Methods for Equilibrium and Fixed point Problems for Nonexpansive Semigroups in Hilbert spaces, J.Optim.Theory.Appl., 146 (2010), No. 2, 491-509.

[9] Combettes, P. L., Hirstoaga, S.A., Equilibrium programming in Hilbert spaces, J. Nonlinear Convex Anal., 6 (2005), No. 1, 117-136. 
[10] Inchan, I., Hybrid extragradient method for general equilibrium problems and fixed point problems in Hilbert space, Nonlinear Anal.Hybrid system, 5 (2011), No. 3, 467-478.

[11] Li, S., Li, L., Su, Y., General iterative methods for a one parameter nonexpansive semigroup in Hilbert space, Nonlinear Anal., 70 (2009), No. 9, 3065-3071.

[12] Li, X. N., Gu, J. S., Strong convergence of modified Ishikawa iteration for a nonexpansive semigroup in Banach spaces, Nonlinear Anal., 73 (2010), No. 4, 1085-1092.

[13] Lin, Q., Viscosity approximation to common fixed points of a nonexpansive semigroup with a generalized contraction mapping, Nonlinear Anal., 71 (2009), No. 11, 5451-5457.

[14] Liu, L. S., Ishikawa and Mann iterative processes with errors for nonlinear strongly accretive mappings in Banach space, J.Math.Anal.Appl., 194 (1995), No. 1, 114-125.

[15] Moudafi, A., Viscosity approximation methods for fixed point problems, J.Math.Anal.Appl., 241 (2000), No. 1, 46-55.

[16] Qin, X., Cho, Y. J., Kang, S.M., Viscosity approximation methods for generalized equilibrium problems and fixed point problems with application, Nonlinear Anal., 72 (2010), No. 1, 99-112.

[17] Song, Y., Xu, S., Strong convergence theorems for nonexpansive semigroup in Banach spaces, J.Math.Anal.Appl., 338 (2008), No. 1, 152-161.

[18] Shimizu, T., Takahashi, W., Strong convergence to common fixed points of families of nonexpansive mappings, J.Math.Anal.Appl., 211 (1997), No. 1, 71-83.

[19] Tada, A., Takahashi, W., Strong convergence theorem for an equilibrium problem and a nonexpansive mapping, J.Optim. Theory Appl., 133 (2007), No. 3, 359-370.

[20] Takahashi, S., Takahashi, W., Viscosity approximation methods for equilibrium problems and fixed point problems in Hilbert spaces, J. Math. Anal.Appl., 331 (2007), No. 1, 506-515.

[21] Zegeye, H., Shahzad, N., Strong convergence theorems for a finite family of nonexpansive mappings and semigroups via hybrid method, Nonlinear Anal., 72 (2010), No. 1, 325-329.

[22] Zhang, S., Rao, R., Huang, J., Strong convergence theorem for a generalized equilibrium problem and a k-strict pseudocontraction in Hilbert space, Appl.Math.Mech., 30 (2009), No. $6,685-694$.

[23] Xu, H. K., Iterative algorithms for nonlinear operators, J. London Math. Soc. (2), 66 (2002), No. 1, 240-256. 